\title{
Experimental study on the effect of fracture scale on seismic wave characteristics
}

\author{
Wei Jianxin *, Di Bangrang and Wang Qiang \\ State Key Laboratory of Petroleum Resource and Prospecting, CNPC Key Lab of Geophysical Exploration, China University \\ of Petroleum, Beijing 102249, China
}

\begin{abstract}
In fractured reservoir beds, fracture characteristics affect seismic wave response. Fractured models based on the Hudson's fractured medium theory were constructed in our laboratory by a backfilling technique. For the same fracture density, the variations of the velocity and amplitude of the primary wave and shear wave parallel and perpendicular to the fracture were observed by altering the diameter (scale) of the penny-shaped fracture disk. The model test indicated that an increase of fracture scale increased the velocity and amplitude of the primary wave by about $2 \%$. When the shear wave propagated parallel to the fracture, the velocity of the fast shear wave hardly changed, while the velocity of slow shear wave increased by $2.6 \%$ with increasing fracture scale. The results indicated that an increase of fracture scale would reduce the degree of anisotropy of the shear wave. The amplitudes of slow shear waves propagating parallel and perpendicular to fractures decreased with increasing fracture scale.
\end{abstract}

Key words: Fracture scale, Hudson's fracture theory, primary wave and shear wave velocity, anisotropy, model test

\section{Introduction}

Concerning the research on seismic wave response characteristics in fractured reservoir beds, Hudson's (1980; 1981) fractured medium theory and fracture density equations are generally used to estimate the fracture density of formation. According to the theory, fractures are considered to be thin penny-shaped disks with an aspect ratio far less than 1 . The fracture density equation is $\varepsilon=\mathrm{Nr}^{3} / \mathrm{V}$, where $\mathrm{V}$ denotes the volume of base material, $r$ denotes the radius of the fracture disk and $\mathrm{N}$ is the total number of fracture disks in the base material. This equation shows that if the product of the number of fractures and the cube of the fracture radius stay constant in the same volume, the fracture density will not change. However, in the actual fractured reservoir beds, it is possible that the fracture density may be the same while the fracture scale is different. Such multiplicity reflection on the seismic records is a general concern in geophysical exploration.

There are many papers about the anisotropy in the physical model published in recent years. Zhu (2004; 2006) studied the attenuation in the fractured model by using the Thomsen theory. Chichinina $(2004 ; 2005)$ proposed a theory called "QVOA" (seismic quality factor Q versus offset and azimuth) to study the characteristics of the fractured media, and gave the relationships among the parameters of the anisotropy. Chapman (2003) and Liu (2006) did some research on kinematic and dynamic anisotropy. Moreover, the characteristics of $\mathrm{P}$-waves and $\mathrm{S}$-waves in equivalent

*Corresponding author. email: weijx@cup.edu.cn Received July 19, 2007 media were studied (Ebrom et al, 1990; Tatham et al, 1992; He et al, 2001). The anisotropy was also studied by using 3-D technology (Li et al, 2003; Wang et al, 2007). In our study, we used the equivalent parameter method, which is usually used to study the effect of fracture parameters on seismic wave propagation. The method in laboratory is that low-velocity disks are inserted into base material to simulate the fractured medium (Ass'ad et al, 1992; 1993; Rathore et al, 1995; Wei, 2004), which accords with the actual fracture distribution as well as the Hudson's fractured medium theory. Prior studies were mainly on the qualitative or quantitative analysis of shear wave anisotropy, but the quantitative observation of fracture scale, especially for primary waves has been lacking. In current geophysical exploration, the primary wave is still widely used. Therefore, the experimental data about the effect of fracture scale on the primary wave and shear wave play an important role in exploration for fractured reservoir beds.

Based on the hypothesis about the fractured model in Hudson's fractured medium theory, we constructed a series of simulated fractured models with the same fracture density by altering the fracture disk diameter and the number of fracture disks in each layer and observed the effect of fracture scale on the wave propagation characteristics with the ultrasonic pulse transmission method. The study could provide basic and quantitative experimental data for recognizing fractures correctly.

\section{Construction of fractured models and testing $\mathrm{P}$-wave and S-wave}

\subsection{Construction of fracture models with different}




\section{fracture scales}

The models were constructed with the method of inserting low-velocity round disks into the base material. Based on the Hudson's fracture density equation, we constructed a series of models with different fracture densities. Firstly, the model fracture density was set to be about $7.4 \%$. Secondly, fracture scale and the total number of fractures in each model were altered. Finally, the number of fracture disks in each layer was calculated.

The base material of the models is epoxy resin with good fluidity which becomes hardened after mixing with a curing agent. Its density is $1.18 \mathrm{~g} / \mathrm{cm}^{3}$. The P-wave velocity is $2630 \mathrm{~m} / \mathrm{s}$ and S-wave velocity is $1,200 \mathrm{~m} / \mathrm{s}$. The simulated fractures are low-velocity material which is composite material containing silicon rubber. Its density is $1.09 \mathrm{~g} / \mathrm{cm}^{3}$, $\mathrm{P}$-wave velocity is $1,360 \mathrm{~m} / \mathrm{s}$ and $\mathrm{S}$-wave velocity is zero. Firstly, a big block of fracture material was made (400× $300 \times 30 \mathrm{~mm}$ ) and its density, P-wave velocity and S- wave velocity were measured. Then, thin and long strips with different thicknesses were cut from the big block and disks with different diameters were machined from these strips using a special tool. The thickness of the thin and long ships was uneven, so we must select the range of thickness from $0.08 \mathrm{~mm}$ to $0.17 \mathrm{~mm}$. More than $50 \%$ of the fracture thickness was from $0.12 \mathrm{~mm}$ to $0.14 \mathrm{~mm}$ and the average thickness was $0.132 \mathrm{~mm}$.

We made seven fracture models. One model with no disks was used as the control model. The other six models had different disks diameters and number. All of the models were made with the same method and at the same time, which could reduce the errors caused by the manufacturing procedure. As shown in Fig.1, the models were made layer by layer and the fracture disks were distributed randomly in each layer. The models were made of epoxy resin of the same weight and the fracture-simulating disks were inserted every time a layer of epoxy resin was poured in the mold. There were 34 layers with fracture disks. The thickness of each fractured layer was approximately the same, $0.17 \mathrm{~mm}$. Seven
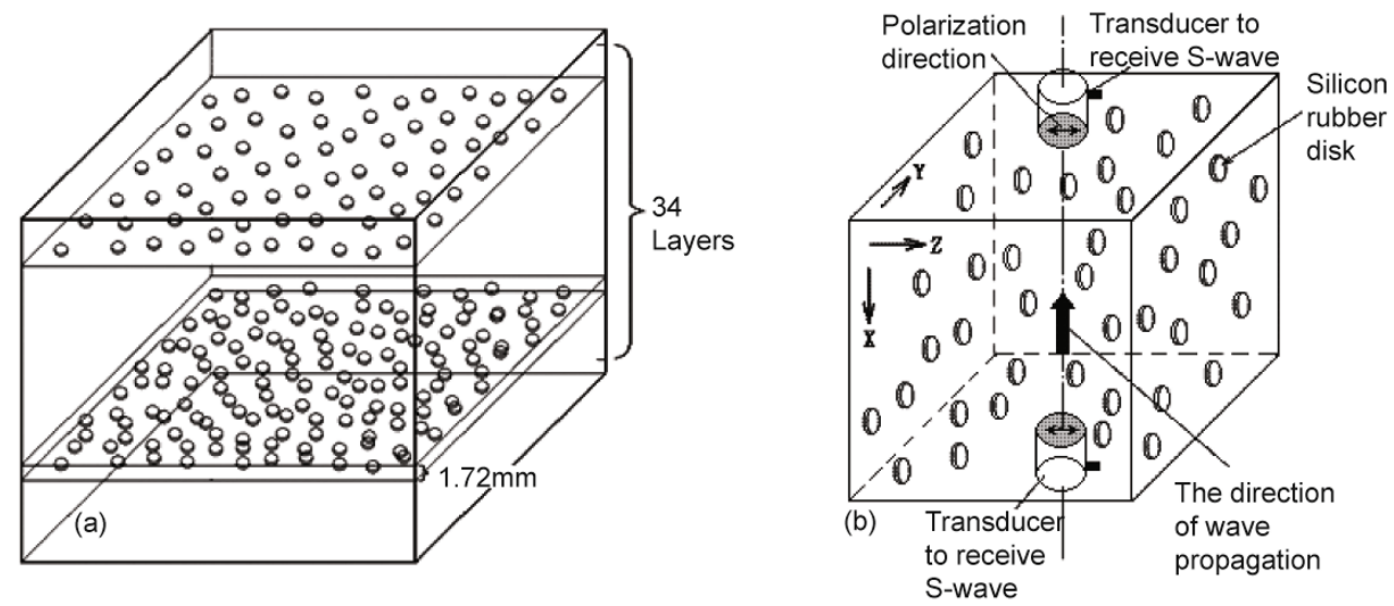

Fig. 1 Fracture distribution of fractured model and testing method

Table 1 Parameters of fractured models with different fracture diameters

\begin{tabular}{ccccccc}
\hline Model & $\begin{array}{c}\text { Fracture } \\
\text { diameter, } \mathrm{mm}\end{array}$ & $\begin{array}{c}\text { Model size } \\
\mathrm{mm}, \mathrm{X} \times \mathrm{Y} \times \mathrm{Z}\end{array}$ & $\begin{array}{c}\text { Volume } \\
\mathrm{cm}^{3}\end{array}$ & $\begin{array}{c}\text { No. of fracture } \\
\text { /layer }\end{array}$ & $\begin{array}{c}\text { Total No. of } \\
\text { fractures }\end{array}$ & $\begin{array}{c}\text { Fracture density } \\
\varepsilon, \%\end{array}$ \\
\hline 0 & 0 & $70.14 \times 70.14 \times 57.3$ & 276 & 0 & 0 & 0 \\
1 & 2.5 & $70.35 \times 69.92 \times 57.32$ & 282 & 313 & 10642 & 7.37 \\
2 & 3 & $70.34 \times 69.92 \times 57.3$ & 282 & 181 & 6154 & 7.37 \\
3 & 3.5 & $70.4 \times 69.86 \times 57.32$ & 282 & 114 & 3876 & 7.37 \\
4 & 4.2 & $70.34 \times 69.9 \times 57.28$ & 282 & 66 & 2244 & 7.38 \\
5 & 5 & $70.38 \times 69.92 \times 57.3$ & 282 & 39 & 1326 & 7.35 \\
\end{tabular}




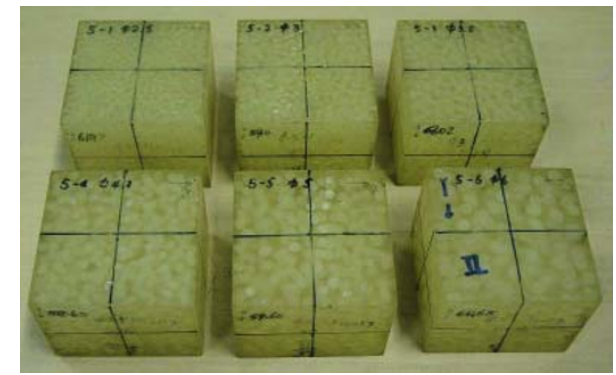

Fig.2 Fracture thickness distribution of different models

models were processed into cubes with the same volume and the three opposite surfaces were smooth and parallel to each other. The parameters of the seven models are given in Table 1. The sixth model's density was $7.37 \%$, a little larger than the other five models. Fig. 2 is a photograph of the seven models. To be convenient for testing and analyzing, we marked coordinates on each model. The $\mathrm{Z}$-axis was vertical to the fracture disk plane, the X-Y plane was parallel to the fracture disk plane and the $\mathrm{X}$-axis was the direction of the largest side.

\subsection{P-wave and S-wave test}

The pulse transmission method was used to test the P-wave and S-wave velocity of the seven models. The source and the transducer to receive $\mathrm{P}$-wave or $\mathrm{S}$-wave were placed on two opposite surfaces in the same direction of the model, as shown in Fig.1(b). P-wave and S-wave ultrasonic transducers were used in this experiment. Both of them had a wide frequency band and short pulse. The S-wave transducer had very good polarization characteristics.

In order to compare the results, the test condition for the seven models was the same. To reduce the testing errors of amplitude, strict coupling was applied. Besides, every model was tested three times and every test had been stacked repeatedly for eight times. The error of $\mathrm{P}$-wave and $\mathrm{S}$-wave velocity was less than $0.5 \%$; the P-wave amplitude error was less than $4 \%$ and the S-wave amplitude error was less than $6 \%$.

The common polarization transmission method (the polarization direction of the transducer to transmit and receive signals is the same) was used in the S-wave test and the S-wave data in two polarization directions parallel and perpendicular to the fracture respectively were recorded.
When the S-wave propagated in the direction of fracture (X or $\mathrm{Y}$ ), the velocity of polarization direction of the S-wave source and receiver parallel to the fracture orientation was called fast shear wave denoted by S1, while the velocity of polarization direction perpendicular to the fracture orientation was called the slow shear wave denoted by S2. When the $\mathrm{S}$-wave propagated in the direction perpendicular to the fracture orientation, the velocity of the S-wave polarization parallel to the $\mathrm{X}$ direction was $\mathrm{Sx}$ and the one parallel to the Y direction was Sy.

Considering the effect of sample load on the transducer's performance, the frequency characteristics of the transducer in the experiment was calculated by observing the waveforms of the model without fracture. Fig 3 gives the waveforms and frequency spectrum curves in the $\mathrm{X}$ and $\mathrm{Z}$ direction since the $\mathrm{Y}$ direction is the same as the $\mathrm{X}$ direction. The $\mathrm{P}$-wave dominant frequency was $194 \mathrm{KHz}$ and the bandwidth was from $106 \mathrm{KHz}$ to $282 \mathrm{KHz}$ in the $\mathrm{X}$ and $\mathrm{Z}$ directions. The S-wave dominant frequency was $130 \mathrm{KHz}$ in the $\mathrm{X}$ direction and $110 \mathrm{KHz}$ in the $\mathrm{Z}$ direction. The data showed that the method of model construction had some effect on the S-wave dominant frequency. Whereas the bandwidth of S-wave was almost the same in the $\mathrm{X}$ direction and $\mathrm{Z}$ direction, which was from $36 \mathrm{KHz}$ to $238 \mathrm{KHz}$.

The $\mathrm{X}$ and $\mathrm{Z}$ velocities of the model without fracture simulation were identical, which showed that the method of model construction did not cause velocity anisotropy. The P-wave velocity was $2,626 \mathrm{~m} / \mathrm{s}$, the $\mathrm{S}$-wave velocity was $1,198 \mathrm{~m} / \mathrm{s}$ in the base material and the testing error was $4 \mathrm{~m} / \mathrm{s}$. The P-wave wavelength was $1.35 \mathrm{~cm}$ and the $\mathrm{S}$-wave wavelength was $1.00 \mathrm{~cm}$, which were estimated from the dominant frequency in Fig.3.

\section{Test results}

As the simulated fracture distribution in the $\mathrm{X}-\mathrm{Y}$ plane was random, only the waveforms in the $\mathrm{X}$ and $\mathrm{Z}$ direction were tested. Fig.4 gives the P-wave test waveforms in the $\mathrm{X}$ and $\mathrm{Z}$ direction of six fractured models. The time of the wave of model with the minimum fracture diameter starting to skip was used as the base line. By connecting the wave's start times of the other five models, it was shown that the P-wave propagation time decreased and the velocity increased with increasing fracture diameter. Table 2 gives the calculated
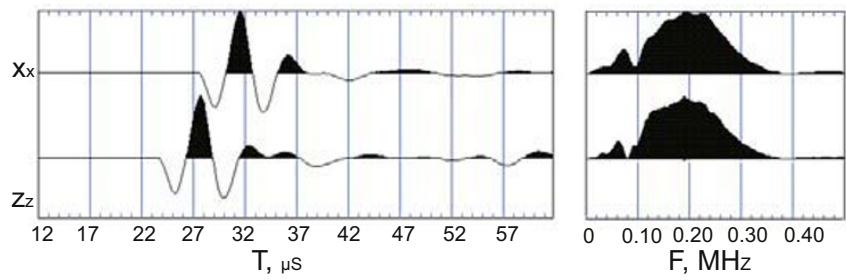

(a) P-wave waveform and frequency spectrum
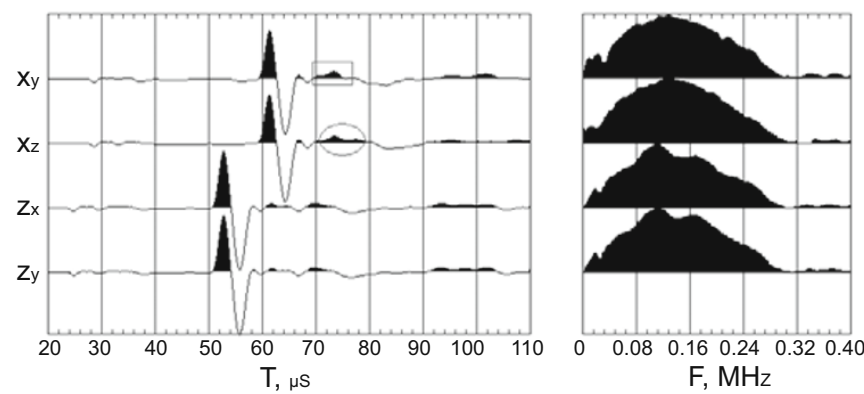

(b) S-wave waveform and frequency spectrum

Fig. 3 P-wave and S- wave recorded in the $\mathrm{X}$ and $\mathrm{Z}$ directions of the 35 layers model without fracture: On the ordinate upper case represents the direction of wave propagation, and lower case represents the S-wave polarization direction 

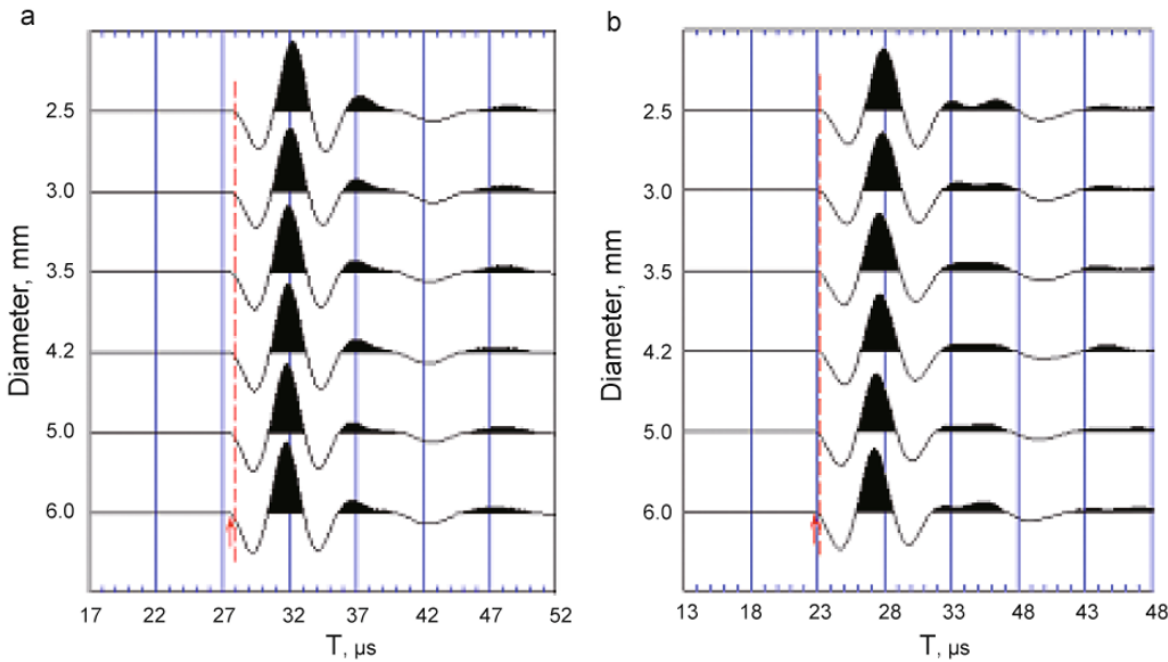

value of each model. The P-wave velocity in the $\mathrm{X}$ direction increased by $37 \mathrm{~m} / \mathrm{s}$ (about $1.4 \%$ ), that in the $\mathrm{Z}$ direction increased by $57 \mathrm{~m} / \mathrm{s}$ (about $2.2 \%$ ), and the error was less than $0.5 \%$. The increase in velocity in the $\mathrm{X}$ and $\mathrm{Z}$ directions was possibly due to different fracture diameters.

Fig. 4 also shows that the fracture scale had little effect on the P-wave amplitude. The curves of $\mathrm{P}$-wave amplitude in the $\mathrm{X}$ and $\mathrm{Z}$ direction changing with fracture scale are shown in Fig. 5. With increasing fracture scale, the amplitude increased a little and the maximum change was less than $8 \%$. It was noticeable that except for the model with the minimum

Fig. 4 P-waves recorded in X-and Z- axis from models with different fracture diameters

Table 2 P-wave and S-wave velocities in X-axis and Z-axis of models with different fracture diameters

\begin{tabular}{|c|c|c|c|c|c|c|c|c|c|}
\hline \multirow{2}{*}{ Model } & \multirow{2}{*}{$\begin{array}{c}\text { Fracture } \\
\text { diameter } \\
\mathrm{mm}\end{array}$} & \multirow{2}{*}{$\begin{array}{c}\mathrm{Vp}-\mathrm{X} \\
\mathrm{m} / \mathrm{s}\end{array}$} & \multirow{2}{*}{$\begin{array}{c}\mathrm{Vp}-\mathrm{Z} \\
\mathrm{m} / \mathrm{s}\end{array}$} & \multicolumn{2}{|c|}{ Vs-X, m/s } & \multicolumn{2}{|c|}{ Vs-Z, m/s } & \multirow{2}{*}{$\begin{array}{c}y-X \\
\%\end{array}$} & \multirow{2}{*}{$\begin{array}{c}\gamma-Z \\
\%\end{array}$} \\
\hline & & & & S1 & S2 & SX & SY & & \\
\hline 1 & 2.5 & 2577 & 2559 & 1181 & 1094 & 1137 & 1133 & 7.37 & 0.3 \\
\hline 2 & 3 & 2586 & 2570 & 1183 & 1106 & 1141 & 1137 & 6.53 & 0.4 \\
\hline 3 & 3.5 & 2588 & 2582 & 1186 & 1113 & 1142 & 1142 & 6.16 & 0 \\
\hline 4 & 4.2 & 2596 & 2592 & 1187 & 1117 & 1141 & 1141 & 5.90 & 0 \\
\hline 5 & 5 & 2607 & 2605 & 1189 & 1121 & 1146 & 1151 & 5.72 & 0.4 \\
\hline 6 & 6 & 2614 & 2616 & 1190 & 1123 & 1151 & 1146 & 5.63 & 0.4 \\
\hline
\end{tabular}

fracture diameter, the amplitude of the other five models increased with increasing fracture diameter and the trend was almost the same in the $\mathrm{X}$ and $\mathrm{Z}$ directions.

When the $\mathrm{S}$-wave propagated in the $\mathrm{X}$ direction of the fractured model, with the change of polarization direction it would split into fast S-wave (polarization direction parallel to the fracture) and slow S-wave (polarization direction perpendicular to the fracture). The fast and slow S-wave records of the six models are given in Fig. 6. In Fig. 6(a), the start times of the fast S-waves are almost the same, which is about $60 \mu \mathrm{s}$. It means that fracture scale almost had no effect on the propagation time of fast S-wave. For slow S-wave, with increasing fracture scale, the start time decreased from $64.4 \mu \mathrm{s}$ to $62.4 \mu \mathrm{s}$. S-wave velocities of each model are shown in Table 2 . When the fracture density was $7.37 \%$, with increasing fracture scale, the maximum change of fast S-wave velocity in the X direction was $9 \mathrm{~m} / \mathrm{s}$ and for slow S-wave velocity, it was $29 \mathrm{~m} / \mathrm{s}$. In other words, when

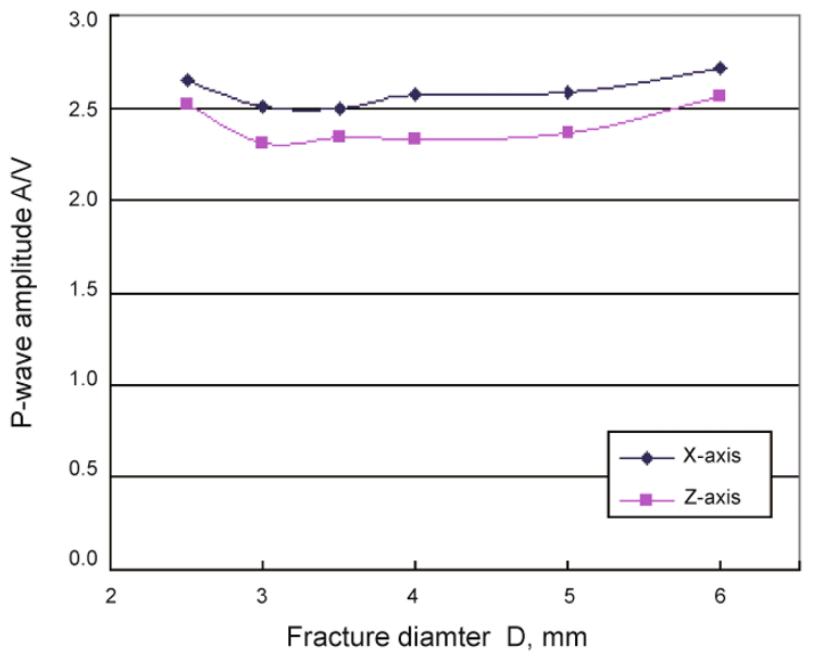

Fig. 5 The curves of P-wave amplitude changing with fracture thickness in X-and Z- aixs 


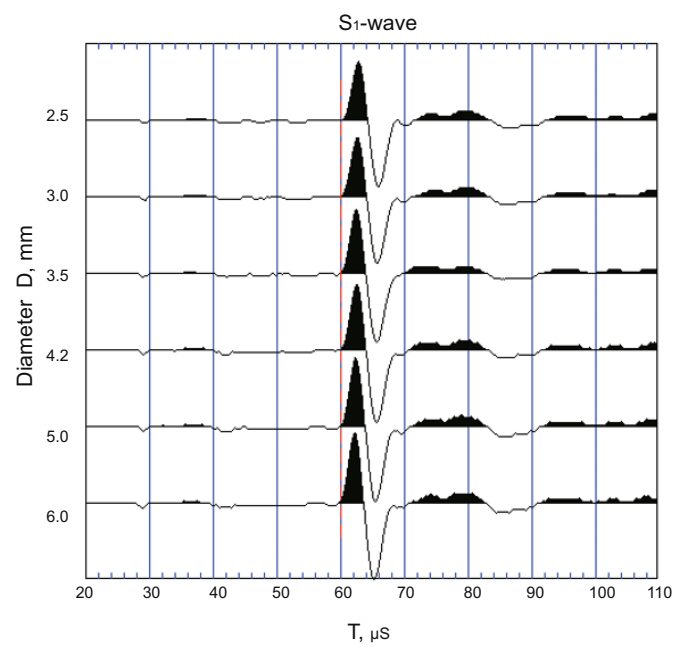

a) Polarization parallel to fractures

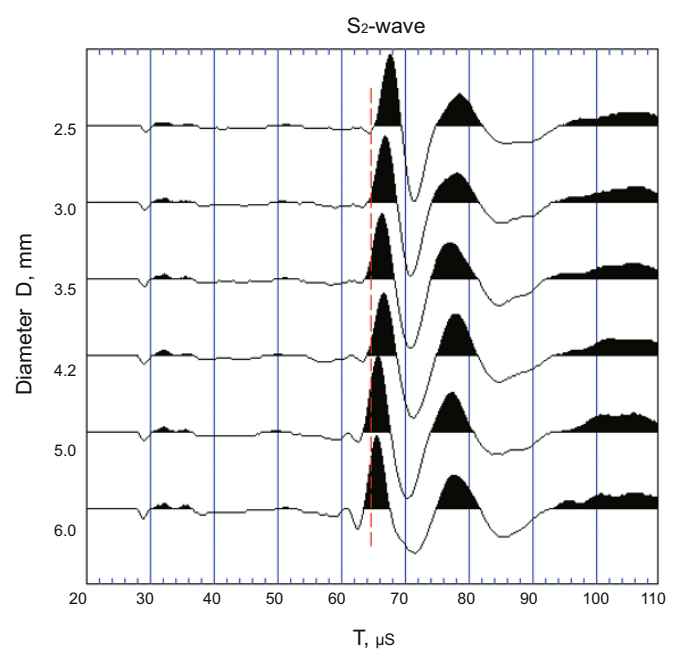

b) Polarization perpendicular to fractures

Fig. $6 \mathrm{~S}$-waves recorded in X-axis of models with different fracture diameters

fracture diameter was $2.5 \mathrm{~mm}$, the velocity difference between fast and slow S-wave was $87 \mathrm{~m} / \mathrm{s}$ and when fracture diameter increased to $6.0 \mathrm{~m} / \mathrm{s}$, the difference decreased to $67 \mathrm{~m} / \mathrm{s}$. This kind of decrease in velocity was mainly caused by the change of fracture diameter.

In Fig. 6(b), it is noticeable that when fracture diameter was larger, a small wave appeared at the start time of slow S-wave and it became larger with increasing fracture diameter. This affects picking the start time of the slow S-wave, but the correct start time can still be picked up by referring to the time change of the largest peak of the waveform.

As the fracture model was symmetrical to the $\mathrm{Z}$-axis, $\mathrm{S}$-wave did not split in the $\mathrm{Z}$ direction. From Table 2 , the velocity change in the two polarization directions was approximately the same. The $\mathrm{S}$-wave records in the $\mathrm{Z}$ direction are given in Fig. 7. It shows that no matter how the S-wave polarization direction changed, with increasing fracture diameter, the velocity increased a little, which was about $14 \mathrm{~m} / \mathrm{s}$.

The curves of S-wave amplitude changing with fracture scale in the $\mathrm{X}$ and $\mathrm{Z}$ directions are given in Fig. 8. When $\mathrm{S}$-wave propagated parallel to the fracture, the amplitude of fast $\mathrm{S}$-wave increased a little with increasing fracture scale. For the change of slow S-wave amplitude, it was different in the first half and second half periods. The amplitude increased in the first half period but decreased faster in the second half period, so in total the amplitude decreased. This kind of change can be seen in Fig. 6(a). The waveform of the second half period becomes wider with increasing fracture scale, and the amplitude becomes relatively smaller. When $\mathrm{S}$-wave propagated perpendicular to the fracture, the effect of fracture scale on S-wave amplitude was evident. In Fig. 8(b), when fracture scale increases by 2.4 times, S-wave amplitude decreases by $46 \%$.

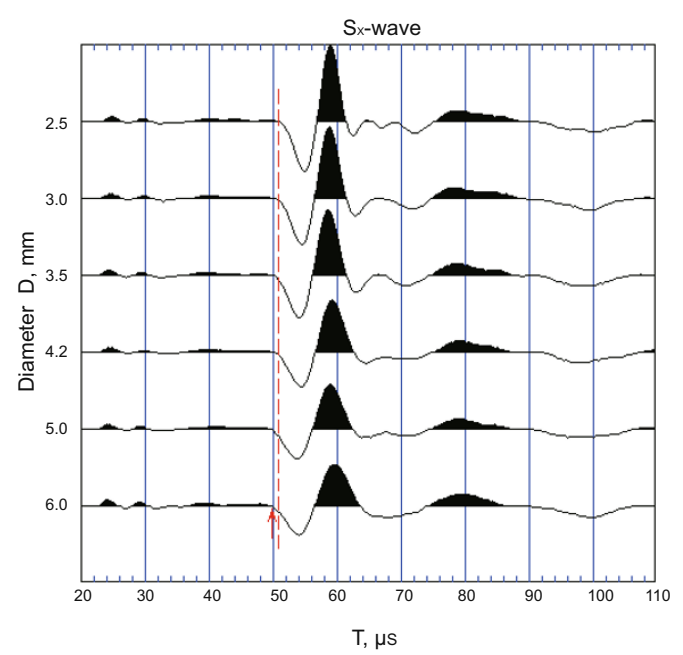

(a) Polarization parallel to $X$-axis

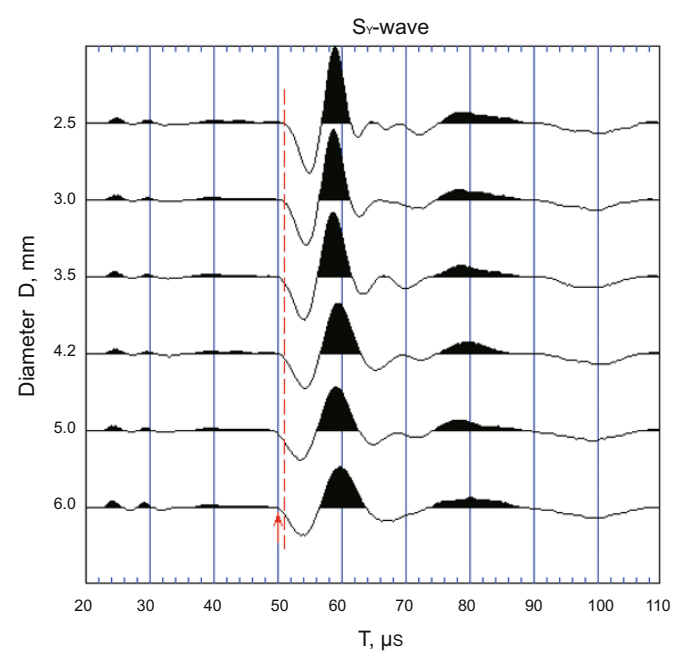

(b) Polarization parallel to $Y$-axis

Fig. 7 S-wave record in Z-axis of models with different fracture diameters 


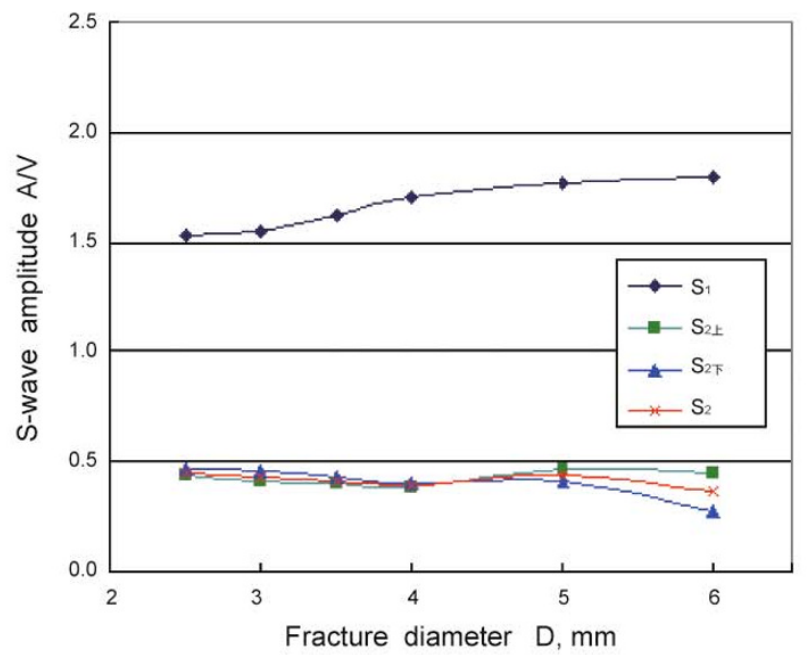

(a) S-wave amplitude in X-axis

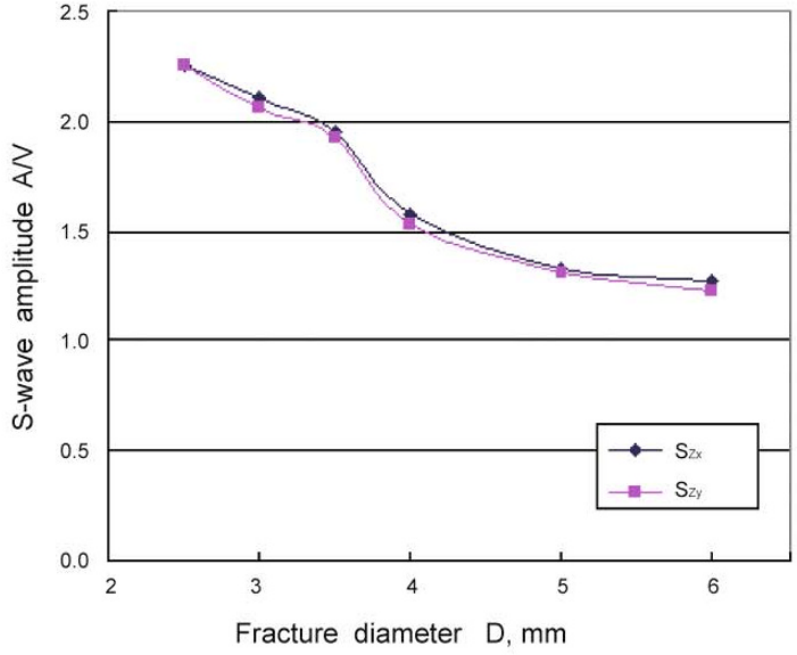

(b) S-wave amplitude in Z-axis

Fig. 8 The curves of S-wave amplitude changing with fracture thickness in X-axis and Z-axis

\section{Discussions}

From Table 2, the curves of P-wave and S-wave velocities changing with fracture diameter can be plotted in Fig. 9. With increasing fracture scale, $\mathrm{P}$-wave and $\mathrm{S}$-wave velocities increased to different extents. P-wave velocity increased by $37 \mathrm{~m} / \mathrm{s}$ in the $\mathrm{X}$ direction and by $57 \mathrm{~m} / \mathrm{s}$ in the $\mathrm{Z}$ direction and the relative changes were $1.4 \%$ and $2.1 \%$ respectively. These changes were bigger than the testing error of the P-wave. In the $\mathrm{X}$ direction, the relative change of fast $\mathrm{S}$-wave was smaller, which was only $0.8 \%$, but for slow S-wave, it was $2.6 \%$. The tracture density of the six models was almost the same, so the change of velocity was related to fracture diameter. This can be explained by the definition of fracture density and energy scattering.

From the research with the same fracture diameter, it is known that P-wave velocity decreases with increasing fracture density. This kind of trend is consistent with the change of velocity with pores in porous media. That is to say, the velocity increases only when fracture density decreases. In this experiment, fracture density was related to product of the fracture number in the same volume and the cube of the fracture radius. The filling volume of fractures in the same volume is related to fracture area and thickness. There is an obvious difference when the fracture radius is small, an increasing ratio of low velocity material filled into the same volume causes the decrease of velocity. This also indicates that the fracture density equation of Hudson's theory may overrate the fracture density of fractured medium. However, the shape of fracture with the aspect ratio much less than 1 is a special case of pores. Although the ratio of fracture filling in the volume affects the wave velocity, the degree of the effect
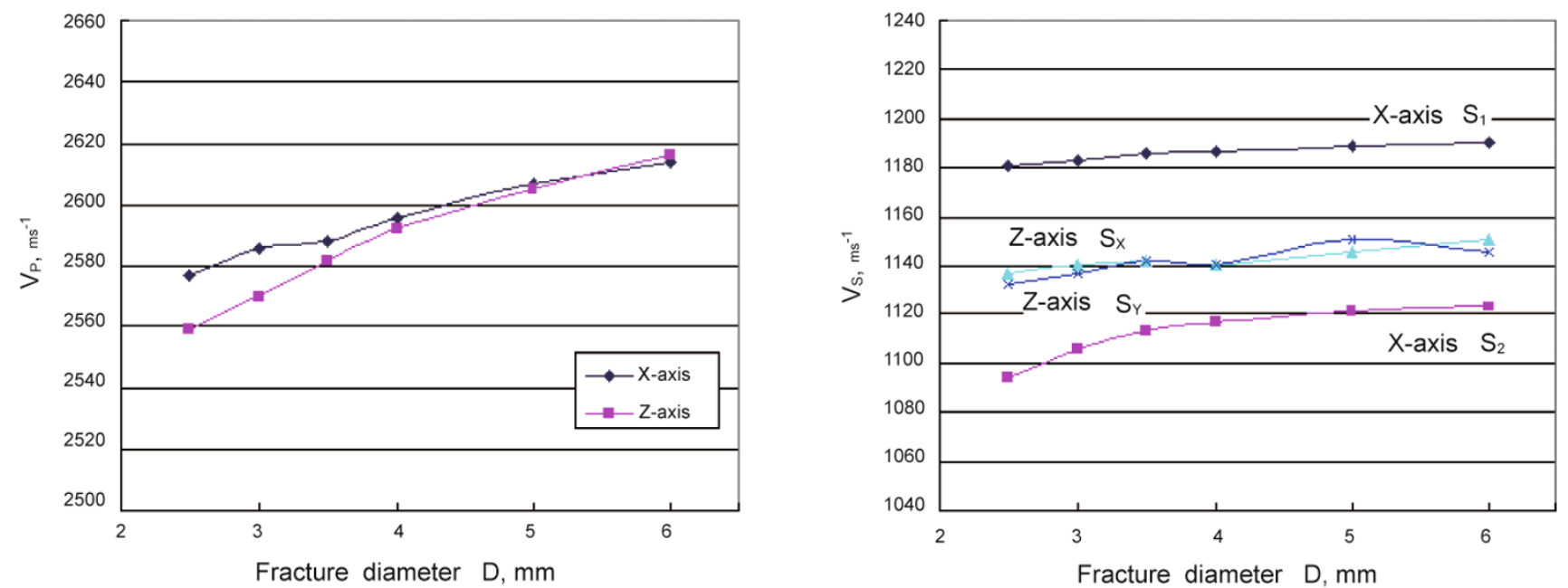

Fig. 9 Curves of P-wave and S-wave velocities changing with fracture diameter 
is quite different from the porous media.

In fractured medium, the effect of fracture scale on a P-wave propagating perpendicular to the fracture is bigger than that parallel to the fracture. Besides, the increase of fracture scale does not affect the decrease of P-wave energy, as the increase of fracture scale reduces the scattering of the wave. When fracture scale is large, there is only one more fracture inside one wavelength. When the fracture scale is small, there is three to four fractures inside one wavelength. This explanation is also reasonable for S-wave propagating in the $\mathrm{X}$ direction.

The effect of fracture scale on the slow S-wave is quite obvious, especially in the direction parallel to the fracture. With increasing fracture scale, the degree of anisotropy parallel to the fracture decreases. The a nisotropy of the S-wave decreased by $24 \%$ when fracture scale increased by 2.4 times. The main reason for the decrease of S-wave anisotropy was the increase of slow S-wave velocity. This indicated that in a fractured medium, the existence of tiny fractures was the main factor which can cause anisotropy. The effect on slow $\mathrm{S}$-wave waveform was mainly on the later vibration which was obvious in the second half period. It was because the increase of fracture scale postponed the restoration of the shear vibration of S-wave.

\section{Conclusions}

Based on the fracture density equation of Hudson's theory, six models with different fracture diameters (scales) and the same fracture density were constructed. P-wave and S-wave parallel and perpendicular to the fracture were measured and the results are as follows:

1) In the models with different fracture scales, the propagating velocity of both $\mathrm{P}$-wave and S-wave no matter in the direction parallel or perpendicular to the fracture increased with increasing fracture diameter. Slow S-wave parallel to the fracture increased the most. This indicated that fracture density equations of Hudson's theory may overrate fracture density in fractured medium.

2) Besides the effect of fracture scale on slow S-wave velocity, the fracture scale affected waveform significantly in the second half period, and this can be used to distinguish the other parameters of fracture.

3) The effect of fracture scale on the P-wave and fast $\mathrm{S}$-wave amplitude was small. However, when the S-wave propagated perpendicular to the fracture plane, the increase of fracture scale affected the amplitude of the S-wave. It decreased by $46 \%$ when fracture scale increased by 2.4 times.

4) The research on fractured model in laboratory is always limited by fracture size and testing frequency. In principle, small fracture diameter should be used. Otherwise the scattering effect of fracture scale on slow S-wave can not be observed when the fracture diameter is smaller than 2.5 times of the base rock wavelength.

\section{Acknowledgements}

This project is supported by the National 973 Plan Project (2006CB202306) and CNPC Applied Theory Study Project (200510101).

\section{References}

Ass'ad J M, Tatham R H and McDonald J A. A physical model study of microcrack-induced anisotropy. Geophysics. 1992. 57(12): $1562-1570$

Ass'ad J M, Tatham R H, McDonald J A, et al. A physical model study of scattering of waves by aligned cracks: Comparison between experiment and theory. Geophysical Prospecting. 1993. 41(3): 323-341

Chapman M. Frequency dependent anisotropy due to meso-scale fractures in the presence of equant porosity. Geophysical Prospecting, 2003, 51: 369-379

Chichinina T. P-wave attenuation anisotropy in fracture characterization: numerical modeling for reflection data. $74^{\text {th }}$ Annual International Meeting, Expanded Abstracts, 2004: 147-150

Chichinina T. QVOA analysis as an instrument for fracture characterization. $75^{\text {th }}$ Annual International Meeting, Expanded Abstracts, 2005: 131-134

Ebrom D A, Tatham R H, Sekharan K K, et al. Hyperbolic traveltime analysis of first arrivals in an azimuthally anisotropic medium: A physical model study. Geophysics. 1990. 55(2): 185-191

He Z, Li Y, Zhang F, et al. Different effects of vertically oriented fracture system on seismic velocities and wave amplitude. Techniques of Physical and Chemical Computation. 2001. 23(1): 1-5 (in Chinese)

Hudson J A. Overall properties of a cracked solid. Mathematical Proceedings of the Cambridge Philosophical Society. 1980. 88: 371-384

Hudson J A. Wave speeds and attenuation of elastic waves in material containing cracks. Geophysical Journal of Royal Astronomical Society. 1981. 64(1): 133-150

Li X Y, Y J, Liu, E, et al. Fracture detection using land 3D seismic data from the Yellow River Delta China. The Leading Edge: 2003. 22: $680-683$

Liu E. Kinematic and dynamic anisotropy. Implication of seismic fracture characterizations. $76^{\text {th }}$ Annual International Meeting, Expanded Abstracts. 2006: 135-139

Rathore J S, Fjaer E, Holt R M, et al. P- and S-wave anisotropy of a synthetic sandstone with controlled crack geometry. Geophysical Prospecting. 1995. 43(6): 711-728

Tatham R H, Matthews M D, Sekharan K K, et al. A physical model study of shear-wave splitting and fracture intensity. Geophysics. 1992.57(4): 647-652

Wang S, Li X Y, Qian Z, et al. Physical modeling studies of 3-D P-wave seismic for fracture detection. Geophysical Journal International. 2007. 168: 745-756

Wei J X. A physical model study of different fracture densities. Journal of Geophysics and Engineering. 2004. 1(1): 70-76

Zhu Y P. Plane-wave propagation and radiation patterns in attenuative TI media. 2004 Annual International Meeting, $74^{\text {th }}$ Annual International Meeting, Expanded Abstracts. 2004: 131-134

Zhu Y P. Effective attenuation anisotropy of layered media. $76^{\text {th }}$ Annual International Meeting, Expanded Abstracts. 2006: 154-158

(Edited by Yang Lei) 